\title{
A DIGITAL RADIO ECHO-SOUNDING AND NAVIGATION RECORDING SYSTEM
}

\author{
by
}

M.R. Gorman and A.P.R. Cooper

(Scott Polar Research Institute, University of Cambridge, Cambridge CB2 1ER, U.K.)

\begin{abstract}
The Scott Polar Research Institute (SPRI) Mk IV $60 \mathrm{MHz}$ radio echo-sounding (RES) system has proven itself to be a most effective and versatile tool in glaciology. During the last 15 years, it has been used from a variety of platforms, both surface and airborne, and over a range of ice thicknesses from $4000 \mathrm{~m}$ to $100 \mathrm{~m}$. However, the photographic recording methods used during this period were felt to be increasingly outdated in the context of modern data handling procedures. Accordingly, in late 1982 the Mk IV system was modified to incorporate fast digitizing of the RES receiver output, with microcomputer-controlled magnetic-tape recording of both the radar data and navigational inputs (Drewry and Liestøl 1985). The new system will be described, along with the improvements in data processing which have resulted from its use.
\end{abstract}

\section{INTRODUCTION}

The fundamental principles of radio echo-sounding are well established. They rely on the fact that polar ice masses are relatively transparent to electromagnetic radiation in the Very High Frequency (VHF) range, c. $30-300 \mathrm{MHz}$. A simple low-frequency radar is flown over the surface, and short pulses are emitted directly downward. These propagate through the air, are partially reflected from the surface of the ice, continue to propagate (with some loss of energy) through the ice mass, and are finally reflected from the ice-bedrock interface. These reflections (together with faint echoes from any internal layering within the ice) return to the radar, where timing of the reflections enables the location of the different interfaces to be determined. By this means, a cross-section through the ice may be built up along any desired track.

Fig.1 is a block diagram of the elements of the complete RES system. The system includes the Mk IV radar, navigation, and recording systems, other avionics, and software for a digital RES system; each will be described in the following sections.

\section{RADAR}

The radar used is the well-proven SPRI Mk IV $60 \mathrm{MHz}$ system, which is a development of the system described by Evans and Smith (1969). The Mk IV system specifications are:

\section{Carrier frequency}

\section{$60 \mathrm{MHz}$}

Pulse length $300 \mathrm{~ns}$

Receiver bandwidth $15 \mathrm{MHz}$

Transmitter power $300 \mathrm{~W}$ peak

System performance $160 \mathrm{~dB}$ (not including antenna gain)

\section{Antenna type}

2 half-wave dipole with reflector

Half-power beamwidth

$90^{\circ}$

Forward gain
A logarithmic receiver provides about $60 \mathrm{~dB}$ of dynamic range. The receiver output is digitized by a fast sample-and-hold circuit, using the sampling scheme discussed below.

The antenna system comprises two half-wave dipoles, one mounted under each wing of the DeHavilland Twin Otter aircraft. In this configuration, the wing acts as a reflector, giving a forward gain of about $8 \mathrm{~dB}$. One antenna is used for transmitting, the other for receiving. Isolation between the two is found to be sufficient $(>40 \mathrm{~dB})$ to make a transmit-receive switch unnecessary.

The radar and recording systems are shock-mounted in a purpose-built aluminium-alloy rack secured in the main cargo compartment of the aircraft.

\section{NAVIGATION SYSTEMS}

The general approach to navigation systems which has been adopted might be termed post hoc. That is, great emphasis is placed on recording sufficient data to accurately reconstruct the precise track of the aircraft after the survey has been completed, rather than attempting to navigate very accurately during the flight. On occasion, however, the facilities provided by modern navigation systems have been used to fly, for example, a pre-determined grid over featureless terrain.

Primary navigation systems of two types have been used with the new system. In 1983, a Doppler navigator, linked through a Tactical Air Navigation System (TANS) navigation computer, provided a continuous read-out of latitude and longitude. Data from the TANS is provided in a serial stream, which is recorded directly on to tape, as described below.

During the 1986 season, a Litton 3000 Omega navigation system was used, which provided a similar data stream for recording. Omega uses low-frequency transmissions from a number of world-wide beacons to obtain a geographic position. However, the accuracy of this system is inferior to the Doppler-TANS combination. The normal accuracy of the Omega system is $2-3 \mathrm{~km}$. This can be significantly improved (by up to an order of magnitude) by the use of along-track position fixes. During the radio echo-sounding operations in mountainous terrain there were frequent opportunities to acquire ground control when crossing nunataks, rock ridges, etc. Vertical aerial photographs were taken to precisely locate the aircraft over such features.

The serial data stream from the primary navigation system (doppler or Omega) includes the following data:

1. Present position (latitude and longitude),

2. Time (GMT),

3. Date (day, month, year),

4. Raw north wind and raw east wind,

5. X-track error,

6. Track angle,

7. True heading,

8. Drift angle,

9. Ground speed, and

10. True air speed.

These data, plus ranges from the Motorola system, compass and air-data outputs, as well as other sensors, such 


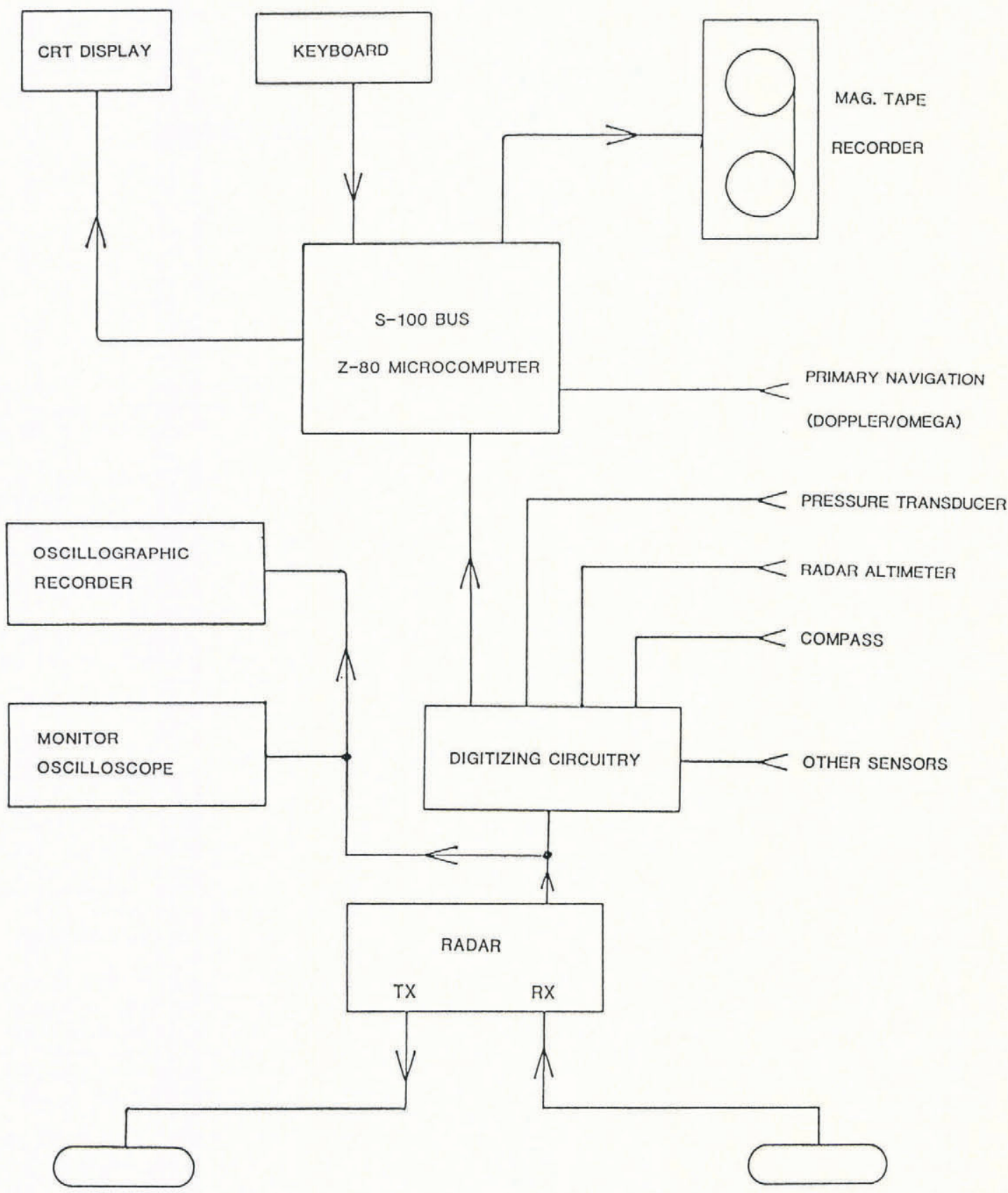

Fig.1. Block diagram of equipment.

as a precise pressure altimeter, are recorded on magnetic tape contiguously with the radar waveform to which they relate. This direct association of radar and navigational data represents a considerable advance over previous systems in which separate records have to be correlated.

Additionally, in 1983, a Motorola Mini-Ranger distance-measuring system was installed in the aircraft, operating in conjunction with ground-based transponders. These were located by satellite geoceiver. This provided much-improved precision over the centre of the Nordaustlandet ice cap, where errors of only $\pm 30 \mathrm{~m}$ are estimated.

\section{OTHER AVIONICS}

Other avionics which are of importance to the RES system include the aircraft radar altimeter and gyrostabilized compass. The radar altimeter is an S-band radar instrument which indicates the terrain clearance, i.e. the vertical clearance between the aircraft and the terrain below. The output of this unit is recorded, and may prove helpful in subsequent data analysis. The same information is usually available from the radio echo-sounder output itself, and there is some indication that the RES terrain clearance figure exhibits lower variance than the aircraft's radaraltimeter output. Nevertheless, the radar-altimeter data provides an important check, especially when flying with small terrain clearances, when the surface return may tend to merge with the tail of the transmit pulse. It is important that an accurate figure for terrain clearance be obtained along the survey flight line, to enable the absolute elevation of the surface to be determined by comparison with the precise pressure-altimeter output.

A five-wire synchro output is available from the aircraft's gyro-stablized compass. Reconstructing the aircraft's heading may be quite important, and magnetic compasses are of little value over much of the polar regions. In practice, the same information is available in the data stream from the primary navigation system (Doppler-TANS or Omega), and this source has proved to be reliable.

\section{RECORDING}

Recording of both radar and navigational data is controlled by an onboard $\mathrm{S}-100$ bus $\mathrm{Z} 80$ based microcomputer, which has proved to be amply powerful. The 
recording media chosen initially were data cartridges of type DC $300 \mathrm{XL}$. These cartridges hold 3.2 Mbytes of data, which represent about $4 \mathrm{~h}$ of airborne recording. For the 1986 season, the recording medium was changed to IBM-compatible magnetic tape of 600 foot $(183 \mathrm{~m})$ reel size. In each case, commercial recorders of the appropriate type were used, communicating with the microcomputer over an RS-232 link in the first instance, and a fast parallel link in the case of the IBM tape. The IBM tape option has greatly eased post-survey data reduction work in the laboratory.

The basic record cycle during airborne work is $2 \mathrm{~s}$. During the first second of each cycle, radar data is gathered, Motorola ranges are acquired, other navigational data read, etc. During the last second, the data are written to tape. Sufficient memory is available to buffer all data with no loss during the required rewind time.

For digitizing the radar output, a sampling scheme was chosen. Using this scheme, the entire received waveform of 256 points is built up during the course of 256 transmit-receive cycles. Fast analogue-to-digital converters are available which could provide all 256 points in the time of one cycle $(60 \mu \mathrm{s})$, but calculations suggest that the advantages to be gained are not worth the added circuit complexity. The aircraft moves only about $0.95 \mathrm{~m}$ during the 256 cycles required for a complete waveform.

The scheme which was chosen samples at $100 \mathrm{~ns}$ intervals in range, to build up a plot of log power against range over $25.6 \mu \mathrm{s}$. This total range has proved to be more than adequate for work in Svalbard. Each sample consists of an 8 bit value, which implies a resolution of about $\pm 0.5 \mathrm{~dB}$ in received power. This is a considerable improvement on the previous photographic recording systems.

In the first version of this equipment, a single average of 8 waveforms, which were uniformly spaced over the $1 \mathrm{~s}$ radar interval, was recorded. Although effective, and with a useful increase of signal-to-noise ratio (c. $9 \mathrm{~dB}$ ), it was realized that information about the variance of the signal was lost by this process. The latest version allows a choice of 2,4 , or 8 waveforms (unaveraged) or the single averaged waveform to be recorded. As the radar waveform comprises about half of each record, there is, of course, a corresponding increase in the volume of data; this will result in a rather short $(c .1 \mathrm{~h})$ recording time per tape for the largest number.

The digitization interval of $100 \mathrm{ns,} \mathrm{compared} \mathrm{to} \mathrm{the}$ frequency content of the data, implies that valid interpolations should be possible between the data points. This was found to be the case, and the leading edge of the strong ice-surface return has been found to be interpolable to an accuracy approaching $2 \mathrm{~m}$. The weaker bottom echoes can also be interpolated, although not to the same accuracy.

The digitized radar data are displayed in the aircraft on a small CRT display, which can be operated in the A-scope mode, or, more usually, in an intensity-modulated mode ( $\mathrm{Z}$-scope). This latter mode provided a very useful display, which appears visually as a cross-section through the ice during the last $8 \mathrm{~min}$ of flying time.

To provide a back-up in case of failure of the digital system, an oscillographic recorder is flown. This provides a $\mathrm{Z}$-scope type of record on heat-developed paper. The dynamic range of this analogue record is rather limited, but it does provide a handy "quick-look" facility, as well as fulfilling a back-up role.

In summary, the new SPRI digital RES represents a considerable advance over previous systems. The direct association of radar and navigational data on the same record, and the directly computer-compatible form of the radar, have greatly eased and speeded data reduction. At the same time, the high density and wide dynamic range of the radar record have made possible investigations (e.g. power reflection coefficient of the bed) which have not previously been attempted on a large scale. An example of a processed digital record is shown in Fig.2.

\section{SOFTWARE FOR DIGITAL RES SYSTEM}

The software installed in the SPRI digital logging systems has been under continuous development since 1983 and has markedly increased in complexity and sophistication
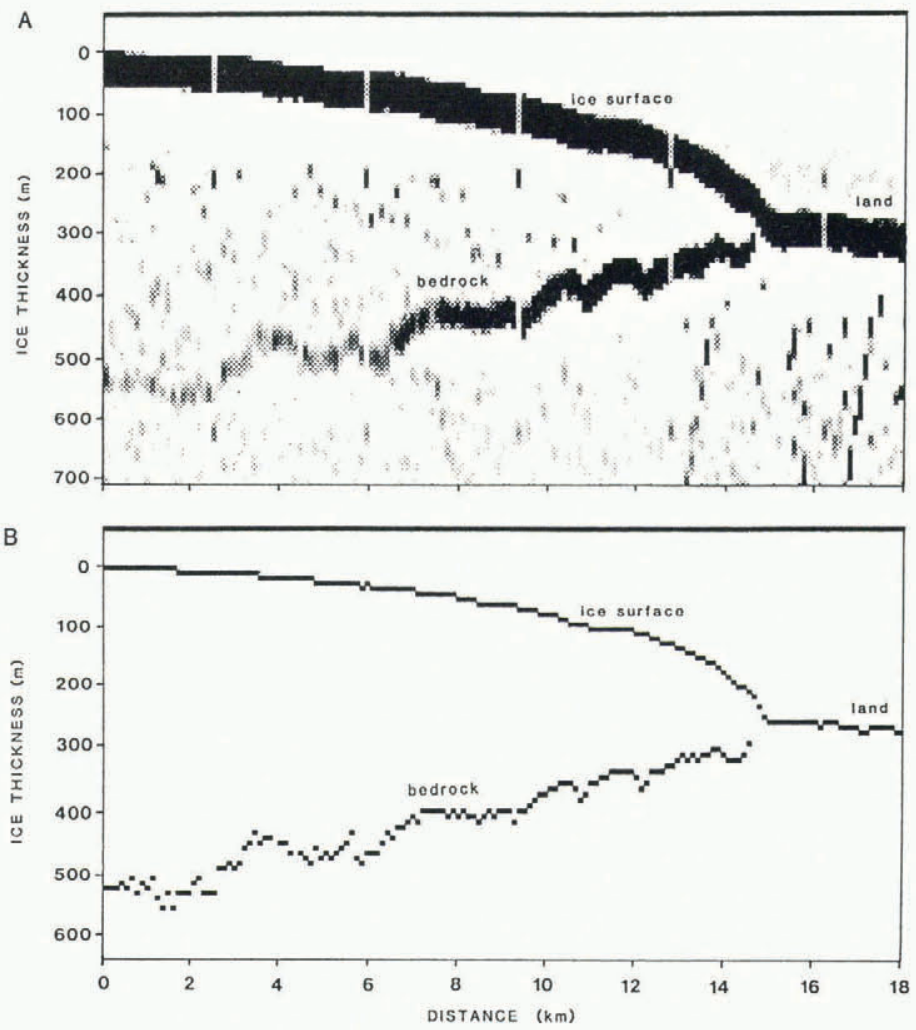

Fig.2. Differentiated digital data.

during this time. It is written in $\mathrm{Z} 80$ assembly language, both to ensure that the program executes as rapidly as possible, and also to overcome the constraints imposed by the limited space available in the Erasable Programmable Read-Only Memory (EPROM) for the program. All versions have had a similar basic design philosophy, in that all consist of a simple loop which continuously checks if data are available in the output buffer and, if they are, dumps them to an output device. The radar digitizing system, and in later versions other devices as well, cause this basic loop to be interrupted. The routine(s) servicing these interrupts read data from the input devices and place them in the output buffer. This basic interrupt-driven scheme allows data to be gathered as fast as they are available, without time being lost by the software in polling devices to see if data are available.

The keyboard interrupt collects characters from the keyboard into a buffer and, when a carriage return is pressed, passes the contents of this buffer to a simple command interpreter. The interpreter is written so as to allow new commands to be included in a straightforward way, giving greater flexibility of use. The current command set includes commands to alter the monitor display, to change the number of A-frames recorded at each radar interrupt, and to fire an external device either on command or at pre-set intervals.

The radar interrupt is the central part of the program, because it causes data to be assembled into a record in the output buffer, as well as reading data from the radar digitizing system, and other inputs such as a pressure transducer. If requested, the software will average the 8 A-frames digitized during the $1 \mathrm{~s}$ of the digitizer's activity. Otherwise, the number of A-frames that have been requested are placed in the output record.

The timer interrupt occurs every $10 \mathrm{~ms}$, and is used to time the firing of an external device which during the current field season was a vertical camera. The camera was used for navigation and to acquire stereo pairs of photographs for studies of surface roughness.

The final interrupt is generated by the aircraft's Omega navigation system each time a byte of data is available. These data are screened to remove that part of the data stream which is not required. The remainder is assembled into a buffer ready for inclusion in the output record. 
Because of the highly modular nature of the software, it should prove to be a straightforward task to modify it for future field seasons, when different data inputs are available.

\section{REFERENCES}

Drewry D J, Liestøl O 1985 Glaciological investigations of surging ice caps in Nordaustlandet, Svalbard, 1983. Polar Record 22(139): 359-378

Evans S, Smith B M E 1969 A radio echo equipment for depth sounding in polar ice sheets. Journal of Scientific Instruments (Journal of Physics E) Ser 2, 2: 131-136 